\title{
Shakeoff of the Vacuum Polarization in Quasimolecular Collisions of Very Heavy Ions*
}

\author{
Gerhard Soff, Joachim Reinhardt, Berndt Müller, and Walter Greiner* \\ Institut für Theoretische Physik der Universität Frankfurt, Frankfurt am Main, Germany \\ (Received 19 November 1976)

\begin{abstract}
The theory of direct electron-positron pair production in the collision of heavy ions is
\end{abstract} \\ formulated in the framework of the quasimolecular model. The pair production process \\ acquires a collective nature for $\left(Z_{1}+Z_{2}\right) \alpha>1$ and can be understood as the shakeoff of the \\ strong vacuum polarization cloud formed in the quasimolecule. The total cross section \\ is, e.g., $76 \mu \mathrm{b}$ for $\mathrm{Pb}+\mathrm{Pb}$ at Coulomb barrier energies.
}

Quantum electrodynamics has up to now been tested only in weak fields $(Z \alpha<1)$ and at short distances between charged particles. For very strong electrostatic fields, if extended over a larger volume of space, a fundamentally new process occurs: the decay of the neutral vacuum into a charged vacuum. ${ }^{1-3}$ This situation can be realized if two heavy nuclei are close together acting combined as a superheavy molecule or atom.

In this Letter we investigate the effect of timevarying, very strong electric fields, which appear during the collision of very heavy nuclei. This leads to another new effect of quantum electrodynamics, namely a collective type of electron-positron creation, due to the coherent action of the strong, extended time-dependent electric field.

Electron-positron pair production (like electron excitation) in heavy-ion collisions is best described in the framework of the quasiadiabatic molecular model. ${ }^{4}$ One starts from a complete set of the stationary two-center Dirac equation ${ }^{5}$

$$
E_{i}(R) \varphi_{i}(\overrightarrow{\mathrm{x}}, R)=H(\overrightarrow{\mathrm{x}}, R) \varphi_{i}(\overrightarrow{\mathrm{x}}, R) .
$$

The functions $\varphi_{i}$ depend on the internuclear dis tance $R$ as a continuous parameter. (In principle, the $\varphi_{i}$ depend on the vector $\vec{R}$, but we neglect here the effects of the orientation of the internuclear axis for the reasons discussed by Betz et $\left.a l .{ }^{6}\right)$ The time-dependent collision is described in the semiclassical approximation by introducing a definite time dependence $R=R(t)$. The part of the time evolution operator not prediagonalized by Eq. (1) is then expressed as ${ }^{7}$

$$
i \dot{R}(\partial / \partial R)=i(\partial / \partial t)-H(R(t)) \text {. }
$$

The initial electron configuration is specified by a set $F$ of occupied states, where $F$ includes all states of the negative energy continuum (definition of the vacuum) and all occupied bound states. ${ }^{8}$ The state vector of this configuration will be denoted by $|F\rangle$. During the collision, electron-positron configurations are excited. Since Eq. (2) is a single-particle operator, we can restrict the basis in first order to one-electron-one-positron excitations. The time-dependent state vector of the system can then be written as

$$
|\Psi(t)\rangle=c_{0}(t)|F\rangle+\sum_{j \in F} \sum_{i \in F} c_{i j}(t) b_{i}{ }^{\dagger} d_{j}^{\dagger}|F\rangle,
$$

$b_{i}{ }^{\dagger}$ and $d_{j}{ }^{\dagger}$ being creation operators for particles and holes, respectively. The normalization condition is

$$
\left|c_{0}\right|^{2}+\sum_{j \subset F} \sum_{i \notin F}\left|c_{i j}\right|^{2}=1
$$

In first-order perturbation theory, the amplitudes $c_{i j}$ are given by

$$
c_{i j}(T)=-\int_{-\infty}^{T} d t\left\langle\varphi_{i}(R)\left|\hat{R} \frac{\partial}{\partial R}\right| \varphi_{j}(R)\right\rangle \exp \left\{i \int_{-\infty}^{t} d \tau\left[E_{i}(R(\tau))-E_{\cdot_{j}}(R(\tau))\right]\right\}
$$

This expression differs from the conventional formulation of pair production in an external electric field. Bang and Hansteen, ${ }^{9}$ e.g., consider the incident particle as the source of a perturbing potential which provides the energy necessary for pair creation. In this way, the pair production is connected with the imaginary part of the photon propagator. In our formulation, however, the total zero-frequency part of the electromagnetic potential is diagonalized in the molecular basis. Therefore, the standard methods of quantum field theory in the interaction picture do not apply. In order to obtain a representation of the pair production process which is independent of the specific basis functions $\varphi_{i}$, we 
introduce the time-dependent density matrix

$$
\rho_{i}\left(\overrightarrow{\mathrm{x}}, t, R(t) ; \overrightarrow{\mathrm{x}}^{\prime}, t^{\prime}, R\left(t^{\prime}\right) \equiv R^{\prime}\right)=\varphi_{i}^{+}(\overrightarrow{\mathrm{x}}, R) \varphi_{i}\left(\overrightarrow{\mathrm{x}}^{\prime}, R^{\prime}\right) \exp \left[i \int_{t}^{t}, d \tau E_{i}(R(\tau))\right]
$$

One verifies by straightforward calculation that

$$
\left|c_{i j}(\infty)\right|^{2}=-\int_{-\infty}^{\infty} d t \int_{-\infty}^{\infty} d t^{\prime} d^{3} x d^{3} x^{\prime}\left[\dot{R} \frac{\partial}{\partial R} \rho_{i}\left(\overrightarrow{\mathrm{x}}, t, R ; \overrightarrow{\mathrm{x}}^{\prime}, t^{\prime}, R^{\prime}\right)\right]^{+}\left[\left[\dot{R}^{\prime} \frac{\partial}{\partial R^{\prime}} \rho_{j}\left(\overrightarrow{\mathrm{x}}, t, R ; \overrightarrow{\mathrm{x}}^{\prime}, t^{\prime}, R^{\prime}\right)\right] .\right.
$$

The total number of excited particle-hole pairs $N_{\mathrm{ph}}$ after the collision is obtained by summing over $i$ and $j$. We introduce the density matrices $\rho_{+}$of occupied (positron) and $\rho_{-}$of vacant (electron) states by

$$
\rho_{+}\left(\overrightarrow{\mathrm{x}}, t, R ; \overrightarrow{\mathrm{x}}^{\prime}, t^{\prime}, R^{\prime}\right)=\sum_{j \in \boldsymbol{F}} \rho_{j}, \quad \rho_{-}\left(\overrightarrow{\mathrm{x}}, t, R ; \overrightarrow{\mathrm{x}}^{\prime}, t^{\prime}, R^{\prime}\right)=\sum_{i \in \boldsymbol{F}} \rho_{i},
$$

the charge-symmetrized density matrices $\tilde{\rho}=\frac{1}{2}\left(\rho_{+}-\rho_{-}\right)$, and $\rho=\frac{1}{2}\left(\rho_{+}+\rho_{-}\right)$. The integrals in Eq. (7) can be interpreted as trace in the coordinate representation, and we can write in matrix formulation:

$$
\begin{aligned}
N_{\mathrm{ph}}=\sum_{j \in F} \sum_{i \in F}\left|c_{i j}(\infty)\right|^{2} & =\operatorname{Tr}\left\{\left[\dot{R} \frac{\partial}{\partial R} \rho_{-}\left(R, R^{\prime}\right)\right]^{+} \dot{R}^{\prime} \frac{\partial}{\partial R^{\prime}} \rho_{+}\left(R, R^{\prime}\right)\right. \\
& =\operatorname{Tr}\left\{\left[\dot{R} \frac{\partial}{\partial R} \tilde{\rho}\left(R, R^{\prime}\right)\right]^{+} \dot{R}^{\prime} \frac{\partial}{\partial R^{\prime}} \tilde{\rho}\left(R, R^{\prime}\right)\right\}-\operatorname{Tr}\left\{\left[\dot{R} \frac{\partial}{\partial R} \rho\left(R, R^{\prime}\right)\right]^{+} \dot{R}^{\prime} \frac{\partial}{\partial R^{\prime}} \rho\left(R, R^{\prime}\right)\right\} .
\end{aligned}
$$

The last form of Eq. (9) clearly exhibits the nature of the process: the shakeoff of vacuum polarization and of bound electrons. Namely, $\widetilde{\rho}$ can be decomposed into the vacuum polarization charge density matrix and a contribution from the bound electrons:

$$
\tilde{\rho}=\frac{1}{2}\left[\sum_{E_{i}<-m} \rho_{i}-\sum_{E_{i}>-m} \rho_{i}\right]+\sum_{\substack{i \in F \\ E_{i}>-m}} \rho_{i}
$$

The term involving $\rho$ is independent of the initial electron configuration and serves to regularize the divergent expression involving $\tilde{\rho}$. The meaning of Eq. (9) is illustrated in Fig. 1: The usual two-photon pair production diagram ${ }^{10}$ is shown in Fig. 1(a); the process described by the theory outlined in this paper is depicted in Fig. 1(b). Because $\left(Z_{1}+Z_{2}\right) \alpha>1$ in the collision of very heavy nuclei, infinitely many interactions with the combined electric field of both nuclei must be taken into account. In this sense, Fig. 1(b) stands for an infinite series of Feynman diagrams like Fig. 1(a). By coherent summation of these diagrams the virtual photon field acquires a collective nature. For $Z_{1}+Z_{2} \ll \alpha^{-1}$ this collectivity is small, meaning that the pair production is well described by diagram 1 (a). However, for $\left(Z_{1}+Z_{2}\right) \alpha>1$ the collective effect becomes dominant. This is expressed in the $Z$ dependence of the total cross section: The lowest-order diagram [Fig. 1(a)] increases as $Z^{4}$, whereas in the superheavy region at constant ion velocities the dependence is approximately $Z^{n}$, with $n \sim 18$. This immediately sheds light on the average number of photon interactions in the production process.

To evaluate Eq. (5) numerically, it is useful to integrate by parts, yielding

$$
\left|c_{i j}(\infty)\right|=2 \int_{0}^{\infty} d t \frac{\cos \left(E_{i}-E_{j}\right) t}{\left(E_{i}-E_{j}\right)^{2}}\left[\ddot{R}+\dot{R}^{2} \frac{\partial}{\partial R}\right]\left\langle\varphi_{i}\left|\frac{\partial H}{\partial R}\right| \varphi_{j}\right\rangle .
$$

We have applied standard methods ${ }^{6,11}$ to replace the $\partial / \partial R$ matrix element. For $R(t)$ we have prescribed Coulomb trajectories. It is sufficient to keep only states with angular momentum $j=\frac{1}{2}$ (i.e., $\kappa= \pm 1$ ) in Eq. (11) because matrix elements with higher angular momentum states are smaller by at least one order of magnitude. This is analogous to vacuum polarization in heavy atoms. ${ }^{12}$ For these states the matrix elements peak strongly for small values of $R$. Therefore, they can be calculated in the monopole approximation where a blow-up nucleus of radius $\frac{1}{2} R$ is substituted for the true two-center potential. The error was shown to be small up to $R \sim 400 \mathrm{fm}$ in an earlier Letter. ${ }^{6}$ Larger values of $R$ do not contribute significantly to the pair production (less than $2 \%$ ). Integration of Eq. (9) over impact parameters yields the final cross section

$$
\sigma_{e^{+} e^{-}}=2 \pi \int_{0}^{\infty} b d b \sum_{\kappa} \int_{-\infty}^{-m} d E \int_{m}^{\infty} d E^{\prime}\left|c_{E^{\prime} E}(b)\right|^{2} .
$$

Table I shows the total pair-production cross section for symmetric collisions with a total nu- 


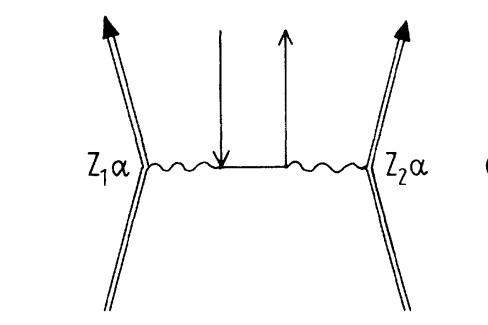

(a)

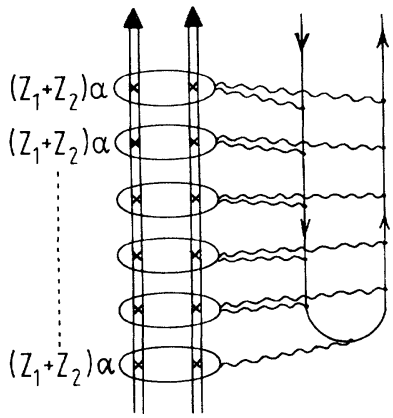

(b)

FIG. 1. (a) The two-photon Feynman diagram describing pair production. There is a second diagram with the electron and positron lines interchanged. (b) The multiphoton diagram representing the collective nature of pair production out of quasimolecular states.

clear charge $Z=Z_{1}+Z_{2}$, at ion energies so that the distance of closest approach is always $16 \mathrm{fm}$. $\sigma_{e^{+} e^{-}}$is seen to increase rapidly with $Z$. The energy spectrum of the created positrons and electrons is shown in Fig. 2(a) for a $\mathrm{Pb}+\mathrm{Pb}$ collision $(Z=164)$. The main difference between the electron and the positron distribution, viz. the behav-
TABLE I. Left, the total pair production cross section $\sigma_{e+e}$ for various superheavy collision systems $Z=Z_{1}+Z_{2}$. The distance of closest approach $(16 \mathrm{fm})$ is kept constant, so that the increase in ion energy contributes somewhat to the sharp rise with $Z$. Right, the cross section for $\mathrm{Pb}+\mathrm{Pb}$ collisions for various ion energies, characterized by the distance of closest approach.

\begin{tabular}{rccr}
\hline \hline$Z$ & $\begin{array}{c}\sigma \\
(\mu \mathrm{b})\end{array}$ & $\begin{array}{c}R_{\min } \\
(\mathrm{fm})\end{array}$ & \multicolumn{1}{c}{$\begin{array}{c}\sigma \\
(\mu \mathrm{b})\end{array}$} \\
\hline 146 & 5.7 & 25 & 3.0 \\
158 & 33.4 & 20 & 19.4 \\
164 & 76.4 & 16 & 76.4 \\
168 & 135 & 13 & 193.4 \\
\hline
\end{tabular}

ior at small energies, is explained by the Coulomb repulsion of the positron states. The positron distribution peaks at ca. $400-\mathrm{keV}$ kinetic energy with $d \sigma / d E_{p} \sim 0.1 \mu \mathrm{b} / \mathrm{keV}$. Also more highenergy positrons than electrons are produced.

The impact-parameter dependence of pair creation is plotted in Fig. 2(b) up to $b=40 \mathrm{fm} . \quad b P(b)$ peaks at $3 \mathrm{fm}$ and falls off exponentially for larger impact parameters. Thus the shakeoff of the vacuum polarization cloud should be experimentally observable in the collision of very heavy ions. Its study in undercritical systems $(Z \leqslant 170)$ is advisable so that it is not disturbed by the decay of the overcritical vacuum. $\mathrm{Pb}+\mathrm{Pb}$ collisions should be favored, because positron production through internal conversion of Coulomb excited

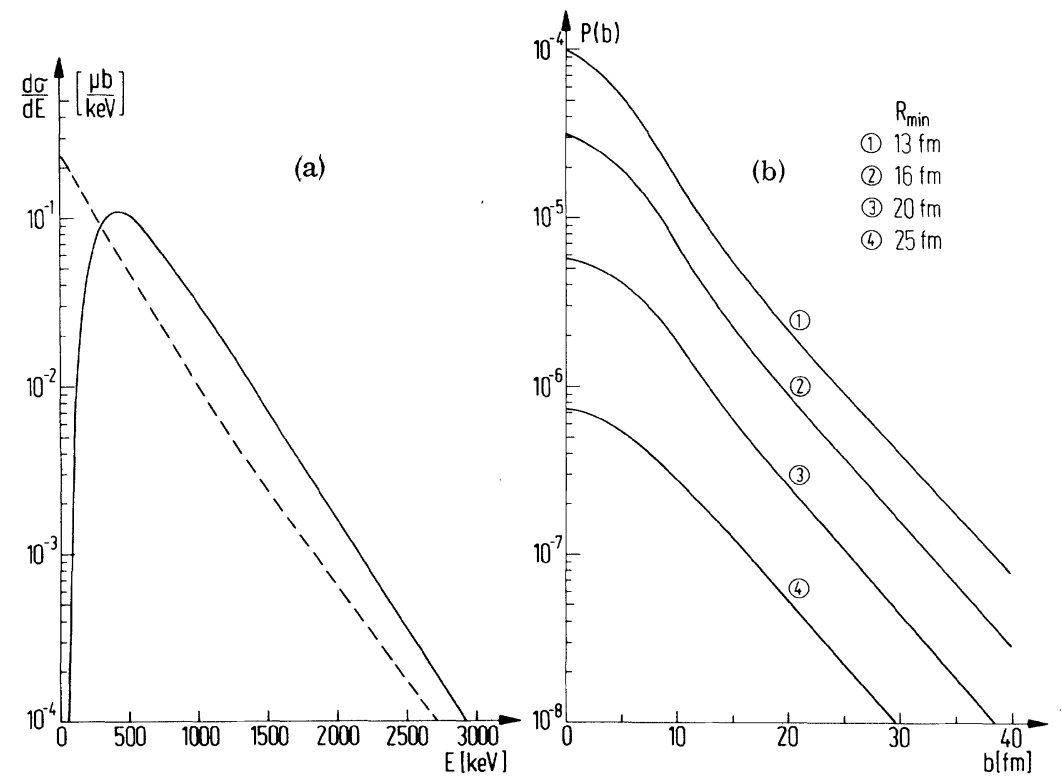

FIG. 2. (a) The positron (solid line) and electron (dashed line) spectrum of pair production in a 1210-MeV (lab) lead-lead collision. (b) The impact-parameter dependence $F(b)$ of pair production in the same collision. 
nuclei should be minimal in this case. The shakeoff of the vacuum polarization cloud, which is a collective type of $e^{+} e^{-}$creation, is a new process of quantum electrodynamics. It is - so to speak - the real setting-free of vacuum polarization charges due to the Fourier frequencies in the collision.

We acknowledge fruitful discussions with Professor J. Greenberg (Gesellschaft für Schwerionenforschung) on the experimental observability of positron production in heavy-ion collisions and are grateful to Professor Werner Scheid, Giessen, for critical reading of the manuscript.

*Supported by the Bundesministerium für Forschung und Technologie and by the Gesellschaft fur Schwerionenforschung.

${ }^{1}$ B. Muller, H. Peitz, J. Rafelski, and W. Greiner, Phys. Rev. Lett. 28, 1235 (1972).

${ }^{2}$ J. Rafelski, B. Mưller, and W. Greiner, Nucl. Phys. B68, 585 (1974).
${ }^{3}$ Ya. B. Zel'dovich and V. S. Popov, Usp. Fiz. Nauk 105,403 (1971) [Sov. Phys. Usp. 14, 673 (1972)].

${ }^{4}$ K. Smith, B. Muller, and W. Greiner, J. Phys. B 8 , 75 (1975)。

${ }^{5}$ B. Mưller, J. Rafelski, and W. Greiner, Phys. Lett. B47, 5 (1973); B. Muller and W. Greiner, Z. Naturforsch. $31 \mathrm{a}, 1$ (1976).

${ }^{6}$ W. Betz, G. Soff, B. Muller, and W. Greiner, Phys. Rev. Lett. 37, 1046 (1976).

${ }^{7}$ The vector potential arising from the nonadiabaticity of the quasimolecule has been neglected. The transverse current $\vec{j}-(4 \pi)^{-1} \nabla \dot{\psi}$ is by a factor $\nu / c \sim 0.1$ smaller than the charge density $\rho$, i.e., the effective magnetic coupling constant is only $Z \alpha v / c$ as compared to the electric $Z \alpha$.

${ }^{8}$ P. G. Reinhard, W. Greiner, and H. Arenhövel, Nucl. Phys. A166, 173 (1971).

${ }^{9}$ J. Bang and J. M. Hansteen, K. Dan. Vidensk. Selsk., Mat.-Fys. Medd. 31 , No. 13 (1959).

${ }^{10}$ V. M. Budnev, I. F. Ginzburg, G. V. Meledin, and

V. G. Serbo, Phys. Rep. 15C, 181 (1975).

${ }^{11}$ D. R. Bates and R. McCarroll, Proc. Roy. Soc. London, Ser. A 245, 175 (1958).

${ }^{12}$ M. Gyulassy, Phys. Rev. Lett. $\underline{33}, 921$ (1974); G. A. Rinker and L. Wilets, Phys. Rev. A 12,748 (1975).

\title{
Positronium Spin Conversion by Phosphorescent Impurities in Gases*
}

\author{
Werner Brandt and Dalia Spektor \\ Department of Physics, New York University, New York, New York 10003
}

(Received 22 October 1976)

\begin{abstract}
Ultraviolet illumination of $\mathrm{Ar}, \mathrm{N}_{2}$, or air containing trace amounts of $\mathrm{SO}_{2}$ or benzaldehyde quenches positronium through interactions with photoexcited impurity triplet states. The measured interaction cross sections are large, $\sim 10^{4} \pi a_{0}^{2}$, and appear to increase as $T^{1 / 2}$ in the range 300 to $400^{\circ} \mathrm{K}$. Triplet concentrations as low as $10^{-8}$ can be detected at atmospheric pressures.
\end{abstract}

We report first measurements of photomagnetic positronium quenching in argon, nitrogen, and air at atmospheric pressures containing small concentrations of benzaldehyde or $\mathrm{SO}_{2}$, which have metastable triplet states. The large cross sections are similar to those observed in solids ${ }^{1}$ and pose intriguing new questions with regard to the quantum theory of excited molecular spinstate interactions with leptonic atoms. This work may have implications for research on gas pollution and, in particular, on $\mathrm{H}_{2} \mathrm{SO}_{4}$ formation via the photodynamic oxidation of $\mathrm{SO}_{2}$ to $\mathrm{SO}_{3}$ in air. ${ }^{2}$

Suppose a gas contains a small concentration, $c$, of molecules or atoms in electronic singlet ground states $(S=0)$ that can be excited by light to populate metastable paramagnetic triplet states $T^{*}(S=1)$ at a steady-state concentration $c_{T} \ll c$. When $o$-Ps (orthopositronium; spin quantum number $S=1$ ) interacts with $T^{*}$, it can be quenched by spin conversion into $p$-Ps (parapositronium; $S=0$ ) with efficiency $\alpha_{o}$, and $p$-Ps quenched into $o$-Ps with efficiency $\alpha_{p}$, by spin flip correlated with the quenching of $T^{*},{ }^{1}$ or possibly by chemical binding of $o$-Ps to the excited molecule. In solids, the $o$-Ps lifetime is only 2 times longer than that of positrons in other states (1 nsec versus $0.5 \mathrm{nsec})$. In gases, it is 100 times longer (100 nsec versus $1 \mathrm{nsec}$ ). Therefore the "positron method" ought to be more sensitive for the detection of photomagnetic impurities through $o-$ Ps quenching in gases than in solids. The results reported here bear this out.

The experiments were performed as follows. A cylindrical copper chamber with length $L$ of 25 $\mathrm{cm}$ and diameter of $10 \mathrm{~cm}$ was closed at one end by a copper plate which held the positron source, consisting of $5 \mu \mathrm{Ci}{ }^{22} \mathrm{Na}$ deposited on an $\mathrm{Al}$ backing. The other end consisted of a quartz window 